\title{
The Relevance of Alpha-1-Acid Glycoprotein in Human Cancer: A Minireview
}

\author{
Nayab Mustansar* \\ Women Medical College, Abbottabad, Pakistan
}

*Corresponding author: Raúl Barrera Rodríguez, Department of Chronic-Degenerative

Diseases, Autoimmunity Laboratory, INER Research Unit, Mexico.

Received Date: June 27, 2019

Published Date: July 02, 2019

\begin{abstract}
PAlpha-1 acid glycoprotein (AGP-1) is a glycoprotein synthesized mainly by hepatocytes. Recently has been speculated that AGP-1 plays an important role in cancer, but its exact biological function is still confusing. Remarkable is the observation that several types of cancer, including hepatic carcinoma, gastric adenocarcinoma, epithelial ovarian cancer, and lung cancer, present an increased expression and secretion of the AGP-1 protein. Furthermore, in some cases, the increase in plasma AGP-1 levels seems to correlate with tumor progression, as they return to normal after chemotherapy treatment. AGP-1 has been allocated as a multifunctional protein with the capacity to modulate the immune response, decrease the chemotaxis and bind chemotherapy compounds, affecting their biodistribution and cytotoxic effectiveness. Because of these capabilities, it has been suggested that AGP-1 could play a role in not only modulate the cancer immune response but have active participation in the surveillance and cancer progression. The accurate evaluation of quantity and quality of AGP-1 in cancer may prove as a new marker can be used to determine the cancer progression or which tumors will respond to which treatments in order to predict the likelihood of drug resistance.
\end{abstract}

Keywords: Alpha-1 acid glycoprotein; Orosomucoid; Cancer; Biomarker; Acute-phase protein

\section{Introduction}

Alpha-1-acid glycoprotein (AGP-1), named as orosomucoid (ORM) is a type of acute-phase protein and is mainly biosynthesized and secreted by hepatocytes $[1,2]$. It is the main component of the fraction of seromucoid proteins at concentrations of between 0.55 to $1.4 \mathrm{mgml}$, but levels increase up to four times compared to baseline as a result of a process of infection or inflammation [3]. AGP-1 is made up of 183 amino acids in a single polypeptide chain with a molecular weight of 41-43 kDa, where multiple N-glycosidic chains contribute up to $45 \%$ with a total weight. AGP-1 exhibits an unusually high degree of glycan branching, being one of the few plasma proteins possessing not only di- and tri-antennary, but also tetraantennary glycans. This degree of branching indirectly results in a high sialic acid composition, which contributes significantly to the unusually low isoelectric point (pI) of the protein, which ranges from 2.8 to 3.8 due to the existence of multiple isoforms $[1,2]$. A number of biological properties have been assigned to AGP-1 over the years. Highlights are the ability to modulate the immune response. In T cells, AGP-1 has the ability to decreases cellular proliferation, induces the production of IL-1 inhibitors, negatively regulate the IL-2, IL-6, and TNF- $\alpha$ production. AGP-1 also has the capacity to inhibit macrophages activation, neutrophil degranulation, platelet aggregation and decrease chemotaxis. As well as its immunosuppressive properties, AGP-1 plays an important role in the perm selectivity of capillary walls, prevents apoptosis, reduces invasion and adhesion to laminin of the cancer cells and has the ability to bind and transport chemotherapy compounds, affecting their volume of distribution cytotoxic effectiveness [4].

Interestingly, some types of cancer can produce and secrete AGP-1, for which it has been suggested this glycoprotein may play an important role in the tumor microenvironment, through influence the immune modulation, physiology, drug resistance, and cancer progression and metastasis. Alterations in the plasma AGP-1 concentrations have been well documented in liver, breast cancer, lung, laryngeal, ovary, urothelial carcinoma [5] and malignant mesothelioma [6]. Remarkably, is a report of Duché JC, et al. which found 2.5 times increase of plasma concentrations of AGP-1 in the breast and lung cancer patient groups while in ovary cancer patients the increase was 1.6 times in relation to healthy population. The AGP-1 concentrations in the cancer population reported in this work ranged between 0.45 and 2.85 gL (mean value 1.1260 .51 gL) [5]. Also, increased AGP-1 concentrations were observed in breast cancer of unknown type and triple-negative breast cancer 
patients in comparison to their controls, which increase with disease progression but to return to normal concentration upon treatment with tamoxifen [7]. Besides, in a study of 100 lung cancer patients, Ayyuba et al. reported high serum levels of AGP-1 in lung squamous cell carcinoma ( $2.93 \mathrm{gl} \pm 1.22$ ) and lung adenocarcinoma $(2.39 \mathrm{gl} \pm 1.13)$ when compared with healthy controls $(0.83 \mathrm{gl} \pm$ 0.21) [8], whereas Fei Li et. al. utilized a cut-off value of 7351.28 ngmg to distinguish infiltrating urothelial carcinoma from bladder cancer patients [9]. On the other hand, an up-regulated expression of AGP-1 was reported in laryngeal cancer patients relative to healthy control group (P0.001). Moreover, its mRNA levels were significantly associated with tumor size, TNM stage, and distant metastasis [10]. According to The Human Protein Atlas (httpswww. proteinatlas.org), a higher level of expression can be detected in liver and prostate carcinoma, whereas the expression levels are variable on different types of cancer (Figure 1a). Experiments of immunoreactivity to AGP-1 in different types of cancer have shown variable results, with tumor cells showing weak to higher cytoplasmic andor nuclear positivity, which seems to depend on the antibody used. Accordingly, several renal cancers, hepatocellular carcinomas along with a few gliomas and ovarian cancers are strongly stained; whereas prostate and pancreatic cancers are mostly negative (Figure 1b) (Figures 1a,1b).

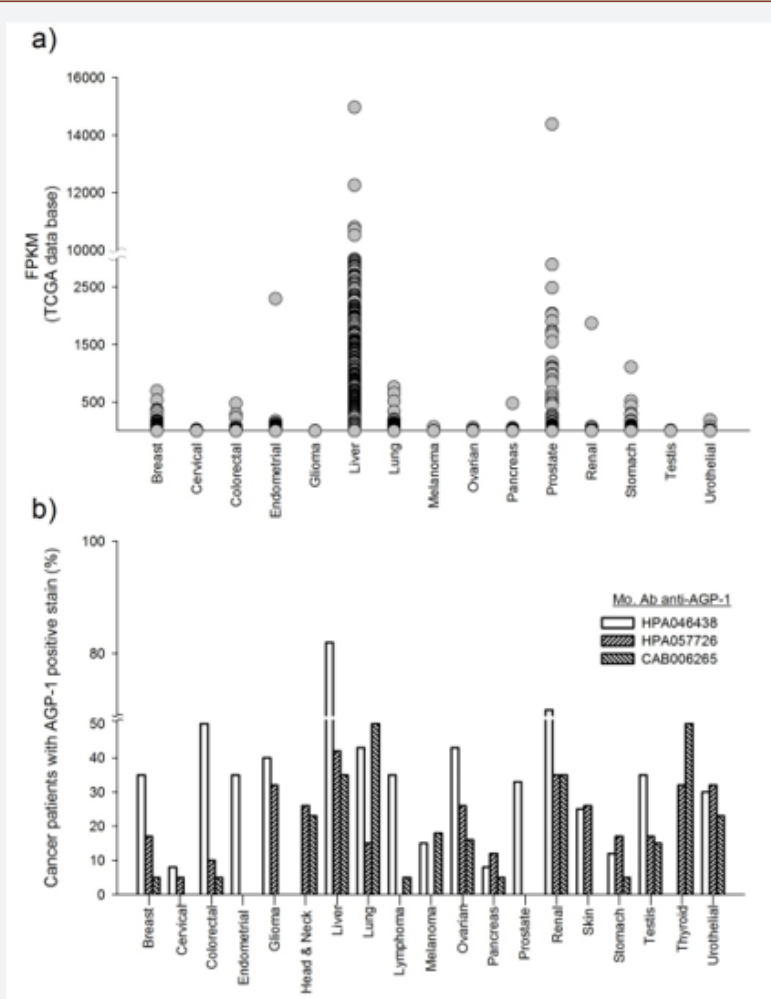

Figure 1: a) Expression analysis of AGP-1 gene in human cancerous tissues. The gene expression levels calculated from the FPKM (Fragments Per Kilobase of transcript per Million) were obtained from RNA-seq data from The Cancer Genome Atlas (TCGA) project (https://www.proteinatlas.org/ ENSG00000229314-ORM1/pathology).

b) Percentage of immunoreactivity against AGP-1 in human cancer samples. The analysis involves three different antibodies that recognize different epitopes. All data were obtained from the Human Protein Atlas (www.proteinatlas.org).
Another important feature that affects AGP-1 functionality is related to the degree of glycosylation. Interestingly, the degree of N-glycosaccharide of AGP-1 was associated with the cancer progression in over $88 \%$ of triple negative breast cancer samples and was inversely correlated with age [7]. Whereas, an increase of $\alpha 1-3$ fucosylated glycoforms of AGP-1 were found in advanced pancreatic ductal adenocarcinoma compared to chronic pancreatitis and healthy controls [11].

\section{Conclusion}

To date, there are several evidences indicated that the prognosis of liver, pancreas, lung and other types of cancer appears to be linked to the AGP-1 levels. However, the significance of AGP1 expression in cancer and their exact biological function is still unclear. Therefore, future investigations could be aimed toward evaluating if alterations in AGP-1 have the potential to be used as biomarker to determine the cancer progression or which tumors will respond to which treatments in order to predict the likelihood of drug resistance.

\section{Acknowledgment}

None.

\section{Conflict of Interest}

No conflict of interest.

\section{References}

1. Fournier T, Medjoubi NN, Porquet D (2000) Alpha-1-acid glycoprotein. Biochim Biophys Acta 1482(1-2): 157-171.

2. Ceciliani F, Lecchi C (2019) The immune functions of alpha1 acid glycoprotein. Curr Protein Pept Sci 20(6): 505-524.

3. Hochepied T, Berger FG, Baumann H, Libert C (2003) Alpha(1)acid glycoprotein: an acute phase protein with inflammatory and immunomodulating properties. Cytokine Growth Factor Rev 14(1): 2534.

4. Luo Z, Lei H, Sun Y, Liu X, Su DF (2015) Orosomucoid, an acute response protein with multiple modulating activities. J Physiol Biochem 71(2): 329-340.

5. Duché JC, Urien S, Simon N, Malaurie E, Monnet I, et al. (2000) Expression of the genetic variants of human alpha-1-acid glycoprotein in cancer. Clin Biochem 33(3): 197-202.

6. Hervé F, Duché JC, Jaurand MC (1998) Changes in expression and microheterogeneity of the genetic variants of human alpha1-acid glycoprotein in malignant mesothelioma. J Chromatogr B Biomed Sci Appl 715(1): 111-123.

7. Dewar, EL (2015) Alpha-1-acid glycoprotein as a potential biomarker of breast cancer in at risk individuals. Thesis (Ph.D.). Edinburgh Napier University, UK.

8. Ayyub A, Saleem M, Fatima I, Tariq A, Hashmi N, et al. (2016) Glycosylated Alpha-1-acid glycoprotein 1 as a potential lung cancer serum biomarker. Int J Biochem Cell Biol 70: 68-75.

9. Li F, Yu Z, Chen P, Guangzheng L, Tieqiu L, et al. (2016) The increased excretion of urinary orosomucoid 1 as a useful biomarker for bladder cancer. Am J Cancer Res 6(2): 331-340.

10. Zhang Y, Wang Z, Bai X, Xu Y (2018) AGP1 acts as a biomarker for diagnosis of laryngeal cancer. Int J Clin Exp Pathol 11(10): 4996-5001.

11. Balmaña M, Giménez E, Puerta A, Llop E, Figueras J, et al. (2016) Increased $\alpha 1-3$ fucosylation of $\alpha$-1-acid glycoprotein (AGP) in pancreatic cancer. J Proteomics 132: 144-154. 